\title{
Methotrexate: should it still be considered for chronic calcium pyrophosphate crystal disease?
}

\author{
Eliseo Pascual ${ }^{1,2^{*}}$, Mariano Andrés ${ }^{1}$ and Francisca Sivera ${ }^{3}$ \\ See related research by Finckh et al., http://arthritis-research.com/content/16/5/458
}

\begin{abstract}
Chronic calcium pyrophosphate crystal arthritis is a clinical consequence of the formation and deposition of these crystals in joints and can result in persistent arthritis. Curative treatment would require the removal of crystals from joints and tissues, but to date all agents tested have proven ineffective. Management of the inflammatory manifestations of chronic calcium pyrophosphate disease includes glucocorticoids, non-steroidal anti-inflammatory drugs, or colchicine, and responses are usually satisfactory. However, in some patients, the response to these agents is poor or they are contraindicated. Methotrexate had been reported as a promising option in small case series; however, in a recent issue of Arthritis Research \& Therapy, a clinical trial failed to confirm the anticipated benefits. Here, we discuss some issues that might have influenced the results of the study, before deciding to abandon methotrexate as a therapeutic option for patients with chronic calcium pyrophosphate arthritis.
\end{abstract}

\section{Editorial}

We thank Finckh and colleagues [1] for their effort in performing a prospective controlled study of methotrexate (MTX) in patients with chronic calcium pyrophosphate (CPP) crystal arthritis, which is a less common clinical consequence of the formation and deposition of these crystals in joints and can result in persistent arthritis usually in older patients. Curative treatment of CPP disease (CPPD) would require the removal of crystals from joints and tissues, but to date all agents tested have proven

\footnotetext{
* Correspondence: pascual_eli@gva.es

'Sección de Reumatología, Hospital General Universitario de Alicante, Pintor Baeza 12, 03010 Alicante, Spain

${ }^{2}$ Cátedra de Medicina (Reumatología), Universidad Miguel Hernández de Elche, Carretera Nacional 332, s/n. 03550 San Juan de Alicante, Alicante, Spain

Full list of author information is available at the end of the article
}

ineffective [2]. Therefore, the management of CPPD relies on the control of the inflammatory manifestations, a scenario in which glucocorticoids, non-steroidal antiinflammatory drugs, and colchicine can work well [3]. However, in some patients, the response to these agents is poor or contraindications for employing them arise, especially as CPPD occurs most often in older patients. Those with persistent disease despite traditional therapy constitute a troublesome subgroup of patients.

MTX seemed a promising option for these patients, as reported in small case series by Chollet-Janin and colleagues [4] and our group [5]. Conversely, other series from France noted no effects [6]. To clarify the effectiveness and safety of MTX, Finckh and colleagues [1] performed this clinical trial, which has failed to confirm the anticipated benefits. Before MTX is abandoned as a therapeutic option for patients with CPPD, it merits analyzing whether any methodological issue might have influenced the results of the trial.

In the study by Finckh and colleagues, they appear to have enrolled two quite different forms of CPPD: some with recurrent (more than three in a 6-month period) episodes of acute arthritis (likely separated by asymptomatic intercritical periods) and others with persistent, polyarthritis-like inflammatory disease. As the authors commented, the size of the study sample is lower than intended, as only 26 pairs were recruited (from the 28-pair calculated sample size) and only seven completed the study, performing an intention-to-treat analysis. Also, the authors selected DAS44 (44-joint disease activity score) as the main evaluation technique, and the sample size was also calculated on the basis of results of previous trials in rheumatoid arthritis (RA). However, around $25 \%$ of enrolled patients had recurrent CPP arthritis, and evaluations at the start of the study or both at the start and the end may have occurred during an intercritical asymptomatic period, hence the low DAS44 results and the lack of change. Only 12 patients showed a polyarticular presentation, the only group where these estimations 
would eventually apply. Therefore, the inappropriateness of the evaluation technique in a group and the low statistical power of the whole study must be taken into account when considering the results of the study.

The diagnosis of CPPD is performed on the basis of consistent clinical features and the identification of the crystals in synovial fluid (SF). In the case of episodes of acute arthritis, the relationship of the crystals with the joint inflammation appears clear, provided that no alternative diagnosis, such as an infection occurring at a joint containing CPP crystals, is reasonable. However, for polyarticular inflammatory disease, the relationship is less clear-cut. Chondrocalcinosis is a common finding in older age groups (approximately 18\% in patients between 75 and 79 years old) [7], and the presence of crystals in SF of previously inflamed joints is constant [8]. In the absence of a negative association between CPP crystals and other diseases, we would expect that one in five patients who initiate a polyarthritis at this age would show chondrocalcinosis on X-rays and likely CPP crystals in their SF. Therefore, the presence of chondrocalcinosis or of a number of CPP crystals in SF in an older patient with persistent polyarthritis and features consistent with those of another disease-such as a seronegative RA (not a rare condition [9]) - might imply the coexistence of the two disorders, RA being the main driver of inflammation; this could explain the possible response to MTX.

The study by Finckh and colleagues also outlines the difficulties of producing evidence in the treatment of less common diseases, especially when there are no economic interests in supporting the research. It also shows the difficulties posed when the clinical presentations are diverse and the reasons for considering them together or apart are not solidly grounded, thus hampering the interpretation of the results. Cross-over designs such as in this trial may facilitate valid conclusions in these circumstances.

We consider that this study should not be taken as definitive, and for those older patients with a seronegative symmetrical polyarthritis showing chondrocalcinosis or CPP crystals (or both) in their SF, MTX still merits a trial.

\footnotetext{
Abbreviations

CPP: chronic calcium pyrophosphate; CPPD: chronic calcium pyrophosphate disease; DAS44: 44-joint disease activity score; MTX: methotrexate;

RA: rheumatoid arthritis; SF: Synovial fluid.
}

\section{Competing interests}

The authors declare that they have no competing interests.

\section{Authors' contributions}

All authors contributed to the drafting of the manuscript and read and approved the final version.

\section{Author details}

${ }^{1}$ Sección de Reumatología, Hospital General Universitario de Alicante, Pintor Baeza 12, 03010 Alicante, Spain. ²Cátedra de Medicina (Reumatología),
Universidad Miguel Hernández de Elche, Carretera Nacional 332, s/n. 03550 San Juan de Alicante, Alicante, Spain. ${ }^{3}$ Sección de Reumatología, Hospital General Universitario de Elda, Carretera Elda-Sax s/n, 03600 Elda, Spain.

Published online: 01 April 2015

\section{References}

1. Finckh A, McCarthy GM, Madigan A, van Linthoudt D, Weber M, Neto D, et al. Methotrexate ethotrexate in chronic-recurrent calcium pyrophosphate deposition disease: no significant effect in a randomized cross-over trial. Arthritis Res Ther. 2014;16:458.

2. Announ N, Guerne PA. Treating difficult crystal pyrophosphate dehydrate deposition disease. Curr Rheumatol Rep. 2008;10:228-34.

3. Zhang W, Doherty M, Pascual E, Barskova V, Guerne PA, Jansen TL, et al. EULAR recommendations for calcium pyrophosphate deposition, Part II: management. Ann Rheum Dis. 2011;70:571-5.

4. Chollet-Janin A, Finckh A, Dudler J, Guerne PA. Methotrexate as an alternative therapy for chronic calcium pyrophosphate deposition disease: an exploratory analysis. Arthritis Rheum. 2007;56:688-92.

5. Andres M, Sivera F, Pascual E. Methotrexate is an option for patients with refractory calcium pyrophosphate crystal arthritis. I Clin Rheumatol. 2012;18:234-6.

6. Doan TH, Chevalier X, Leparc JM, Richette P, Bardin T. Forestier R; French Society for Rheumatology Osteoarthritis Section, Premature enthusiasm for the use of methotrexate for refractory chondrocalcinosis: comment on the article by Chollet-Janin et al. Arthritis Rheum. 2008;58:2210-1.

7. Neame RL, Carr AJ, Muir K, Doherty M. UK community prevalence of knee chondrocalcinosis: evidence that correlation with osteoarthritis is through a shared association with osteophyte. Ann Rheum Dis. 2003;62:513-8.

8. Martinez-Sanchis A, Pascual E. Intracellular and extracellular CPPD crystals are a regular feature in synovial fluid from uninflamed joints of patients with CPPD related arthropathy. Ann Rheum Dis. 2005;64:1769-72.

9. Brasseur JP, Huaux JP, Devogelaer JP, De Deuxchaisnes CN. Articular chondrocalcinosis in seropositive rheumatoid arthritis. Comparison with a control group. J Rheumatol. 1987;14:40-1. 